\title{
Digital Human Modeling for Product Lifecycle Management
}

\author{
H. Onan Demirel ${ }^{1}$ and Vincent G. Duffy ${ }^{1,2}$ \\ ${ }^{1}$ School of Industrial Engineering \\ ${ }^{2}$ School of Agriculture and Biological Engineering \\ Purdue University \\ 47907 West Lafayette, IN, USA \\ hdemirel@purdue.edu, duffy@purdue.edu
}

\begin{abstract}
This paper presents the current and probable future applications of Digital Human Modeling (DHM) in Product Lifecycle Management (PLM) implementations. A Formula 1 race car and a marine vessel were developed by the integration of PLM and DHM software packages. Dassault Systemes' CATIA V5 PLM solution package was used for CAD/CAE design/analysis and UGS Tecnomatix JACK software was utilized for visual/mathematical ergonomics and human analysis. Literature review for future work, [1] and [2], was discussed to investigate the potentials of DHM in PLM for simulation of a blast motion in Navy vessels. The results and observations indicated that integration of DHM and PLM packages have potentials to improve the product development efforts and offer an enhanced approach for simulation of complex systems where there is human-machine integration.
\end{abstract}

Keywords: Digital Human Modeling, Advance Manufacturing, Product Development, Human Factors and Ergonomics, Product Lifecycle Management, Engineering Design, Motion Capture, Simulation \& Modeling.

\section{Introduction}

Use of DHM applications and integration of those with PLM solutions have noticeable advantageous in product development and digital manufacturing. Number of design changes and cost of rapid prototyping can be minimized by DHM and PLM integration [3]. CIMdata's research conclude that on average, organizations using digital manufacturing technologies can reduce lead time to market by $30 \%$, the number of design changes by $65 \%$ and time spent in the manufacturing planning process by $40 \%$. Production throughput can be increased by $15 \%$ and overall production costs can be cut by $13 \%$ [4].

\subsection{Human Element in PLM}

PLM is a strategic business approach which incorporates managerial and engineering aspects of product definition by integrating people, process and information. PLM uses product definition information to manage the entire lifecycle of the product from 
concept creation to end of life by composing a strong collaboration across different segments in the enterprise. PLM applies a consistent set of business solutions in support of the innovative creation, manufacturing, management, distribution and service of products [5]. Complex product development strategies should consider the human-machine integration and ergonomics aspects of the concept product before it's ever launched. One key product development strategy studied in this paper is the utilization of DHM for PLM applications. Many industries have the same challenges where the human element is not being effectively considered in the early stages of the design, assembly, and maintenance of products. However the use of DHM tools can increase the quality by minimizing the redundant design changes and the improve safety of products by eliminating ergonomics related problems [6,7]. An example, at Ford Motor Co. involved the JACK human simulation solution to evaluate ergonomics and worker safety in installing a new satellite digital antenna radio system. This analysis occurred early, at the product design review stage. This reduced the late design modification efforts and helped assess performance and ergonomics based problems prior to prototyping. [8].

\subsection{Need of DHM for PLM}

DHM uses digital humans as representations of the workers inserted into a simulation or virtual environment to facilitate the prediction of performance and/or safety. Also, it includes visualizations of the human with the math and science in the background [9]. DHM helps organizations design safer and efficient products while optimizing the productivity and cost [10]. Applications that incorporate DHM have the potential to enable engineers to integrate ergonomics and human factors engineering principles earlier in the design process [11]. One of the advantages of the DHM applications is that Motion Capture tools can be used to drive the DHM and facilitate reduction of injuries \& comfort prediction through virtual interactive design of work stations and new products [12]. This method allows manufacturers and designers to predict potential risk before production begins [13].

Traditionally DHM applications have been utilized by manufacturing and design industry. One of the first digital human modeling applications was implemented by the U.S. military for cockpit design. Virtual drivers were used to assess the safety and the performance of the vehicles, and the need of expensive physical mockups were minimized. The largest application areas of digital human models are vehicle design, auto and off-road equipment manufacturing [14]. More recently, technological developments and advancement in the CAE tools expands the application areas of DHM. Studies show that one can find current/probable applications of DHM in transportation design, work environment simulation, training and simulation of healthcare personnel and cognitive human modeling.

Design and development of advance products or systems is an iterative process where designs are needed to be modified, changed or reverse engineered due to customer, work or manufacturing demands and constraints. Also, the essential components of a product development; people, departments, equipment and systems can not perform work needed in isolation. There is a definite need for integrating those essential components during design, analysis, production, marketing and service of the products. Integration of DHM and PLM tools may resolve the challenges of 
product development from design to service, and provides control of product development process. This integration also increases the engineering design and analysis capabilities, improves the product ergonomics, enables human-machine virtual reality applications and provides cost and time savings.

\section{Method}

This study examined two product development applications by integration of DHM and PLM tools. A Formula 1 race car and a marine vessel were designed by the integration of PLM and DHM software packages. Dassault Systemes' CATIA V5 PLM solution package was used for CAD/CAE design/analysis and JACK DHM software was utilized for visual/mathematical ergonomics and human analysis [15,16]. Authors' design methodology and DHM strategies of new product development process are explained in detail and figures from design and analysis phases are provided.

\subsection{Formula 1 Race Car Design and Driver Comfort Assessment}

The utilization of DHM for PLM is evaluated by the integration of UGS Tecnomatix Jack, one specific piece of DHM software by UGS, with Dassault Systemes' CATIA V5 PLM software in development of a sample Formula 1 race car. The complete CAD model is designed by using CATIA V5 software. (see Fig.2) The CAD model is composed of 40 constraint-based individual sub-models. Each sub-model is also composed of numerous mechanical parts which are created and then assembled by using CATIA V5's Mechanical Design workbench. Utmost care is taken during constraint based solid modeling in order to construct a valid model with transferability characteristics. Constraint based solid modeling approach brings the advantage of integrating the created model with other CAE software (Fluent, Ramsis, MADYMO) Also, Sectioning and Clash Analysis are applied to visually inspect the parts in assembly. (see Fig.1a.) During CAD model development mechanical integrity of several sub-models are tested by CATIA V5's Generative Structural Analysis.

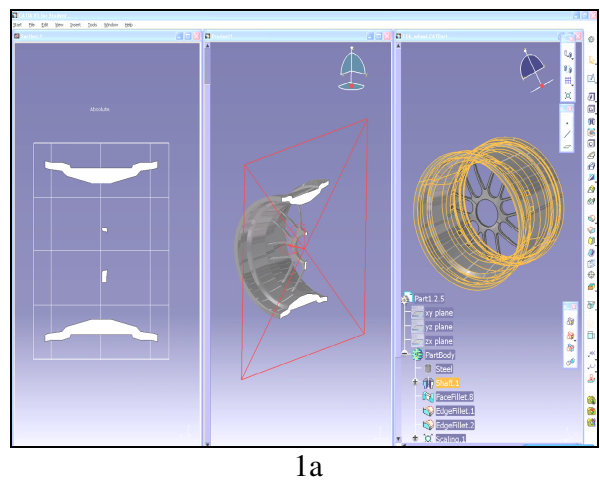

Fig. 1a. Constraint based CAD solid modeling

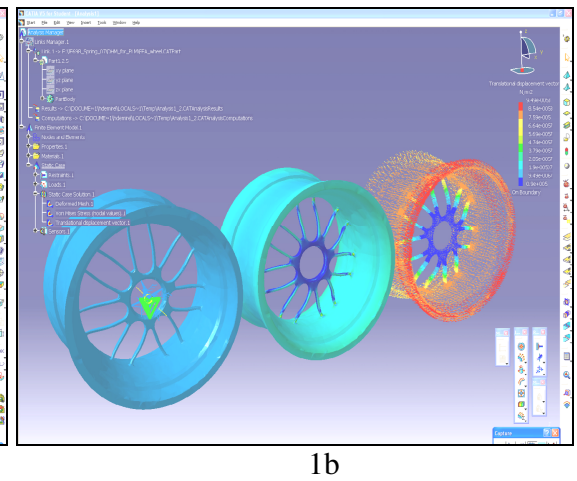

Fig. 1b. Generative structural analysis 
A static analysis case is developed to test the boundary conditions by assigning different scenarios (forces, accelerations, etc.) to check whether the part withstands the effects of applied forces without losing its mechanical integrity. (see Fig. 1b.)

Ergonomics Design and Analysis workbench of the CATIA V5 is utilized to optimize the cockpit space and comfort of the driver. The digital human model is inserted into the Formula 1 race car from built-in anthropometric library and a suitable driver posture is assigned by using Human Posture Analysis workbench. Visual checks (vision, reach envelope, clash detection...) are applied to ensure that driver position is visually legal. (No penetration between human model and race car,

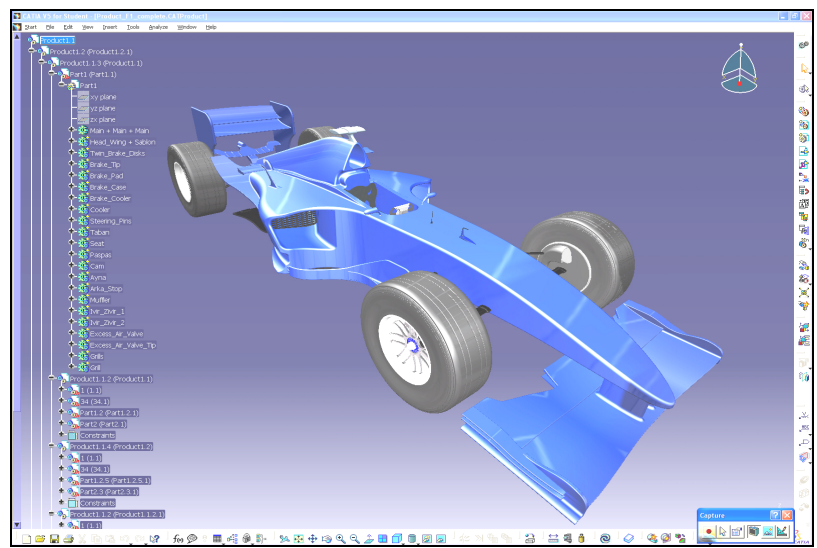

Fig. 2. Final CAD model with Real Time Rendering applied

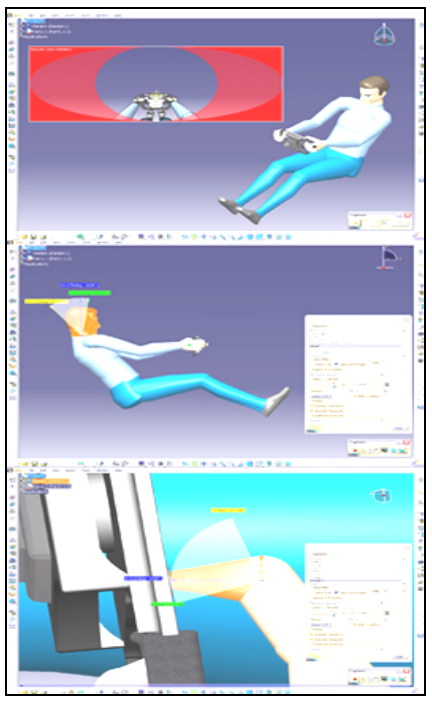

$3 \mathrm{a}$

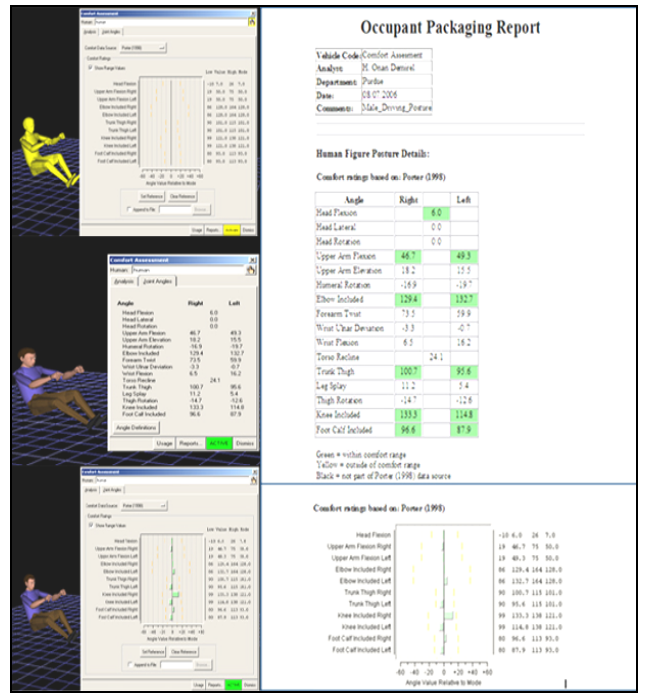

$3 b$

Fig. 3a. DHM Virtual Design

Fig. 3b. Driver Comfort Analysis 
no visual awkward body posture...) (see Fig. 3a.) The given posture and the car then exported to Jack 5 software. The assigned driving posture in CATIA V5 environment is mimicked in Jack 5 software by using built-in Jack human models. The mimicked posture is then analyzed for driver comfort by using Comfort Assessment module under Occupant Packaging Toolkit menu. Comfort Assessment module determines if the assigned posture is within the comfort range based on 3D joint angles and overall position analysis. The Comfort Assessment analysis output highlights if any of the body parts are within/outside the comfort range. (see Fig. 3b.)

Several cockpit features are changed and awkward body positions found are modified until the comfort of the human model and structural integrity of CAD model is matched to the design objectives. This dual validation method, virtual and mathematical, provides a more extensive analysis and offers a better resolution to accommodate optimum driving standards and comfort. After completion of assembly, the complete CAD model is rendered by Real Time Rendering workbench, which enhances the product development attempts by adding almost-real visualization effects (shadows, reflections, etc.) to the model. (see Fig. 2.)

\subsection{DHM in Marine Vessel Design and Simulation}

The product development methodology discussed in Formula 1 race car design can be utilized to resolve the design and analysis challenges in marine applications. The paper discussed [2] indicates that integration of DHM applications may improve the design, analysis and simulation needs of marine vessels.

The paper [2] also indicates that the underwater shock produced by an underwater explosion remains one of the biggest threats to ships and shipboard personnel due to extreme high accelerations in very short duration. Authors used a lumped parameter model which is a simplified mechanical system that divides the human body (sitting upright in a chair) in different segments. The human body is subjected to a vertical shock and the lump parameter model is used to identify the parts of the human body where the impact forces exceed the critical limits. Also, the influences of structural damping and stiffness of the peak loads acting on the human body can be investigated by using this lumped parameter model. [2]

A parallel design and analysis approach similar to the Formula 1 race car is studied to show the potential of DHM and PLM integration with advanced CAE applications in naval vessel design and analysis. Similar to the Formula-1 race car CAD modeling procedure, a ship model is developed from scratch by using CATIA V5. The Generative Shape Design workbench is utilized to sketch the external skin (External hull in CATIA V5 tutorial is utilized) of a naval vessel. After that the external skin is used as the boundary of the deck and transversal bulkhead plates of the base unit. Then, a simplified inner structure of the ship is developed by replicating the base unit by utilizing Structure Object Detailed Design workbench. (see Fig. 4a.) Longitudinal deck plates, transversal bulkhead plates and beams are designed and stiffened to form the skeletal structure of the ship. Mechanical integrity of the external hull is tested by CATIA V5's Generative Structural Analysis. A static analysis case is developed to observe the what-if effects of applied distributed forces on a non-stiffened external hull. (see Fig. 4b.) 


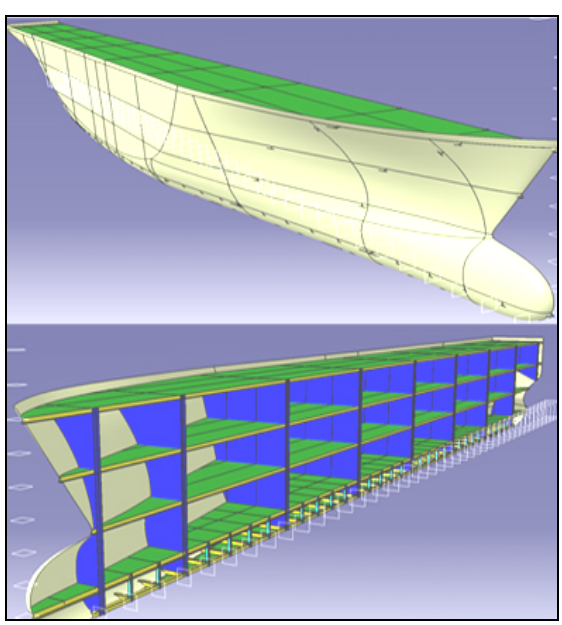

$4 a$

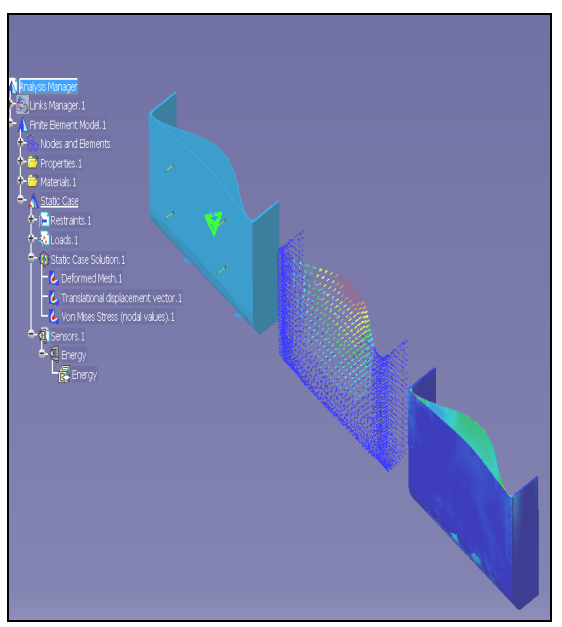

$4 b$

Fig. 4a. Constraint based CAD solid modeling

Fig. 4b. Generative structural analysis

Ergonomics Design and Analysis workbench of the CATIA V5 is utilized simultaneously with CAD construction tools to visually confirm posture, position and visual ratio of the inserted human model respect to ship structure. A door panel is created on one of the transversal bulkhead plates by carefully assessing the inserted human model's anthropometric information. (height, arms span width, etc.) Location of the door (door height from deck plate) and door size is visually fixed in regard to the inserted human model. After the door panel is created, a walking posture is assigned to human model and visual checks (vision, reach envelope, clash detection, etc.) applied to ensure that human model is visually legal. (No penetration between

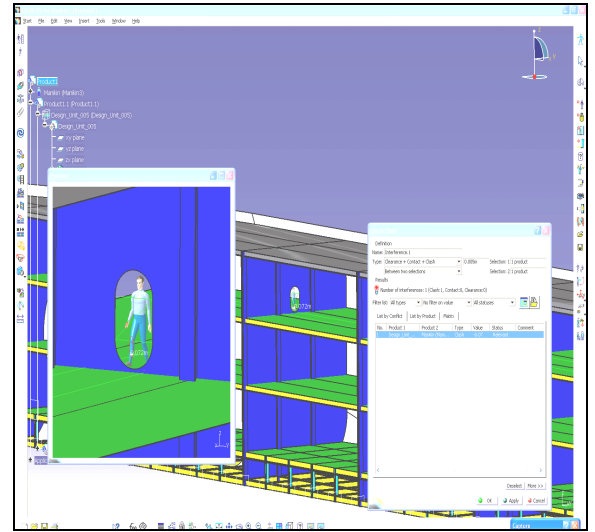

$5 a$

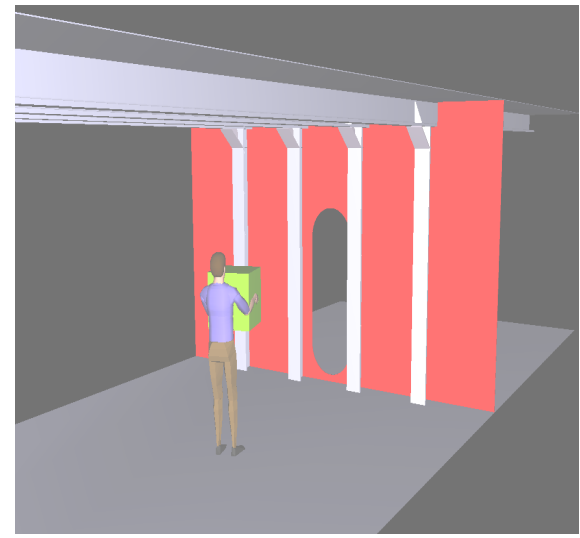

$5 b$

Fig. 5a. CATIA Clash Test

Fig. 5b. JACK PUT task simulation 
human model and panels, no visual awkward body posture) (see Fig. 5a.) The given posture and the design unit are then exported to JACK software. The assigned walking posture in CATIA V5 is mimicked in JACK by using built-in JACK human models. The mimicked posture assigned to JACK model is then inserted into Task Simulation Builder module to simulate a walking task through the door panel. The assigned walking simulation is used to check if the door height and width is blocking the passage. Furthermore, a PUT task simulation with $25 \mathrm{~kg}$ cubic load $(0.5 \mathrm{~m} \times 0.5 \mathrm{~m} x$ $0.5 \mathrm{~m}$ ) is assigned to human model to verify if the walking passage is still valid with assigned load. (see Fig. 5b.)

\section{Results}

CATIA V5 multi-task design and analysis interface gives a great flexibility to user by incorporating complex CAD modeling, generative structural tests and human visual performance tools on a single software package. Throughout the design and analysis development, CAD models are exported to JACK software to assess Driver Comfort Analysis. Depending on comfort analysis, several dimensions of the driver's cockpit is changed to accommodate optimum driving standards and comfort. It was observed that this dual file transfer/editing protocol available between CATIA V5 and JACK integration increased the speed and the quality of product development while reducing time needed for virtual prototyping and redundant changes. The analysis of critical body joints and clash detection between human and race car improved the safety and comfort of the driver. The Occupant Packaging Report shows that the current cockpit design meets the ergonomics, safety and design objectives. In addition, the advance Real-time Rendering workbench of CATIA V5 also enhanced the product development efforts by adding an aesthetic touch to the CAD model.

The literature review and the expertise developed during Formula 1 race car design shows parallel potentials in development of marine vessels by using DHM and PLM tools. The lumped parameter model (multi-degrees of freedom) used by Z.Zong and K.Y.Lam [2] is an improved way over SDF (single-degree-of-freedom) to asses the effects of blast motions in marine vehicles. The simplified mechanical system shows a good approximation of body parts under the stress of underwater shock motions. However further research and development is needed to investigate design, analysis and simulation of complex systems with the aid of advance CAE software applications which incorporate powerful visual and mathematical analysis capabilities. Also, CATIA V5's Structure Detailed System/Object Design module is a powerful tool in ship structure design and user friendly interface eases the design and analysis efforts. Integrating this PLM solution package with dynamic DHM packages may enhance the capabilities of current DHM and PLM implementations.

\section{Conclusion}

PLM approach proved its strength and capabilities by resolving broad engineering and business challenges. DHM have significant impact on the product design and it is able to shorten design-to-built process time and cost [17]. Also, DHM applications help 
engineers to improve ergonomics, safety and human factors in design and product development [15]. The literature review, Formula 1 race car and marine vessel design practices show that integration of DHM and PLM can be a common solution for complex product development challenges. Advance vehicles can be designed and checked for human safety and comfort prior to prototyping and production. Design of complex systems and their interaction with the humans can be simulated through DHM and PLM integration. Overall, DHM and its integration with PLM have potential to improve the product development challenges and provide control of the entire process of designing and analyzing a product before it's ever launched.

Formula-1 race car and marine vessel design discussed in this paper illustrates a product development methodology through concept sketching, solid modeling, mechanical analysis, manufacturing, and marketing under a single frame. One of the most important aspects of the product development challenges studied in this paper is that up-to-date engineering techniques are harmonized with aesthetics skills to demonstrate a versatile marketing opportunities for a profitable product. This advanced integration needs further development and research efforts however studies and applications motivate that the integration between DHM, PLM and CAE tools would be a probable solution for design/analysis and simulation of complex vehicles/systems.

\section{Future Work}

Observations and results presented in this paper were motivated by the need to develop advanced simulation and design methodologies for navy ships. Despite the advantageous of mathematical models discussed in the paper by Z.Zong and K.Y.Lam [2], still more studies are needed to incorporate visual/virtual design components to mathematical models. Previous studies discussed rely on effectiveness of crash test dummies and accelerometers installed in the navy ships and data is collected by livefiring tests. This time consuming tests are raising the questions about safety, cost effectiveness and environmental issues. The elimination of live firing tests by computer simulations have potential to improve the safety, minimize the environmental and financial concerns and reduce the time needed for analysis phase of the design. One possible solution for above challenges can be the integration of DHM and PLM tools with advanced CAE applications which have the capabilities of dynamic analysis of humans in mechanical systems.

Products that are designed and analyzed in this paper rely on the integration between CATIA V5 and UGS Tecnomatix JACK. Both software applications and developed products showed potential improvements in design and analysis of complex systems where human-machine integration is present. However, the demands and constraints of reviewed naval project [2] require a comprehensive approach in utilization of DHM and PLM tools. In order to simulate an underwater shock and to investigate its threats to ship and shipboard personnel, an advanced integration of DHM, PLM and CAE applications with the finite element and dynamic humanmachine analysis capabilities are needed.

One of the industry wide available dynamic human-machine simulation software is the MADYMO, developed by Tass Inc. MADYMO is the worldwide standard for 
occupant safety analysis featuring generic multi-body and finite element capability, a full range of predictive and efficient dummy models and tools to support the restraint optimization process [18]. There are available CAD solid models, Finite Element and Fluid Dynamics models/analysis to observe the effects of the blast motion on naval ships however additional efforts are needed to combine these models with DHM tools to have a comprehensive dynamic human-machine simulation. Integration of MADYMO with available PLM and DHM tools may make this simulation possible. The MADYMO software can be used to simulate complex dynamic response of humans and mechanical systems subjected to impact/blast forces. The paper [1] supported by U.S. Navy demonstrates the capabilities of MADYMO's prediction of response of human in blast-induced blunt trauma by replicating an experimental anthropometric crash test dummies placed in an office space where they exposed to blast forces. Results show that the MADYMO has ability to demonstrate the effects of the blast motions applied and it properly validates human-machine interaction virtually and mathematically [1]. Even though the experiment environment is different (building vs. marine vessel); still there are considerable similarities in simulation development. The ship by itself can be considered as a steel reinforced building where each individual deck beams corresponds to stories. Also, the transverse beam and longitudinal frame of the ship can be considered as columns and arches of a building. Also the biggest application similarity would be each office in building can be thought as compartment in a ship.

Further research and development is needed to resolve the challenges discussed above. A more extensive integration of DHM, PLM and CAE solution packages, (includes dynamic human analysis tools) are demanded to satisfy the comprehensive requirements of complex product design, analysis and simulation needs. However, expertise and motivation gained throughout this study and the literature review demonstrate that technological improvements in CAE tools may enhance this integration and extend the application capabilities of DHM and PLM tools.

\section{References}

1. Bogosian, D., Avanessian, H.D., Ashbery, W.: Analytical and Experimental Studies to Predict Response of Humans to Blast-Induced Blunt Trauma 2001. In: Presented at 10th International symposium of Munitions eith Structures, San Diego, California 2001 (2001)

2. Zong, Z., Lam, K.Y.: Biodynamic Response of Shipboard Sitting Subject to Ship Shock Motion. Journal of Biomechanics 35-43 (2002)

3. Chaffin, D.B.: On simulating human reach motions for ergonomics analyses. Human Factors and Ergonomics in Manufacturing 12, 235-247 (2002)

4. Teresko, J.: Digital Manufacturing: Not Only Manufacturing. Industry Week (November 2006) http://www.industryweek.com/ ReadArticle.aspx?ArticleID=12821

5. CIMdata, What is PLM? CIMdata (2006) http://www.cimdata.com/PLM/

6. Duffy, V.G. (ed.): IE 590D Applied Ergonomics. Spring 2006. Purdue University (November 2006) http://web.ics.purdue.edu/ duffy/index590D

7. Duffy, V.G. (ed.): IE 656 Digital Human Modeling for Design. Spring 2006, Purdue University (November 2006) //web.ics.purdue.edu/ duffy/index656

8. Teresko, J.: Process Planning: Human Simulation. Industry Week (November 2006) http://www.industryweek.com/ReadArticle.aspx?ArticleID=12822 
9. Sundin, A., Ortengren, R.: Digital human modeling for CAE applications. In: Salvandy, G. (ed.): Handbook of Human Factors and Ergonomics, pp. 1053-1078. John Wiley \& Sons, New York (2006)

10. Yang, J., Abdel-Malek, K., Nebel, K.: Restrained and unrestrained driver reach barriers, SAE Technical Paper 2004-01-2199

11. Chaffin, D.B., Faraway, J., Reed, M.B., Woolley, C., Rabourn, R.: From modeling to improved ergonomics in designed systems, 2005, University of Michigan HUMOSIM Laboratory, p. 27 (2005)

12. Li, K., Duffy, V.G., Zheng, L.: Universal accessibility assessments through Virtual Interactive Design. International Journal of Human Factors Modeling and Simulation (in press, 2006)

13. Duffy, V.G.: Using the Virtual Build methodology for computer-aided ergonomics and safety. Human Factors and Ergonomics in Manufacturing (in press, 2006)

14. Reed, M.P.: Research: Digital Human Modeling University of Michigan (2006) http://mreed.umtri.umich.edu/mreed/research_dhm.html

15. UGS Tecnomatix Jack. UGS (November 2006) http://www.ugs.com/products/Tecnomatix/ human_performance/jack/

16. Catia V5 Dassault Systemes (October 2006) www.3ds.com

17. Chaffin, D.B.: Digital Human Modeling for Vehicle and Workplace Design. Society of Automotive Engineers, Warrendale, PA (2001)

18. Tass TNO-MADYMO. MADYMO (January 2007) http://www.madymo.com/cms/ index.php 